\title{
La Loi en panne symbolique ou comment mourir à la singularité devant la Loi de Kafka
}

Loi et cas dans la philosophie polaire du droit : une impossible rencontre?

\section{Alain Papaux}

\section{(2) OpenEdition}

\section{Journals}

Édition électronique

URL : http://journals.openedition.org/ress/462

DOI : $10.4000 /$ ress.462

ISSN : 1663-4446

Éditeur

Librairie Droz

\section{Édition imprimée}

Date de publication : 1 janvier 2006

Pagination : 53-76

ISBN : 2-600-00959-0

ISSN : 0048-8046

Référence électronique

Alain Papaux, "La Loi en panne symbolique ou comment mourir à la singularité devant la Loi de Kafka », Revue européenne des sciences sociales [En ligne], XLIV-133 | 2006, mis en ligne le 10 novembre 2009, consulté le 03 mai 2019. URL : http://journals.openedition.org/ress/462 ; DOI $10.4000 /$ ress.462 


\title{
LA LOI EN PANNE SYMBOLIQUE OU COMMENT MOURIR À LA SINGULARITÉ DEVANT LA LOI DE KAFKA Loi et cas dans la philosophie polaire du droit : une impossible rencontre?
}

\begin{abstract}
AVANT-PROPOS
Fable (fabula $\left.{ }^{1}\right)$ de l'impasse, jeu de dupes du droit, ou plutôt absence totale de jeu de la loi hiératique, de la forteresse-Loi: en peu de mots la nouvelle de F. Kafka Devant la loi.

L'impasse, entendue aussi bien à la manière quasi topologique de Voltaire - le cul-de-sac - qu'en la façon figurée de Diderot - «situation sans issue » -, se peut formuler en termes ontologiques - l'individu (moderne), le solipsisme - et en termes épistémologiques (ou cognitifs) - la position du «singulier» (logique du particulier) éberlué devant le général et abstrait de la «loi ».

Impasse par absence de mesure commune, de «com-mensurabilité»: entre le pôle «loi» et le pôle «homme de la campagne», point de relation; Kafka les juxtapose seulement. Et pour mieux souligner cette absence de lien malgré la porte, la porte ouverte, il dispose un gardien devant la porte, devant chaque porte. Et que fait un gardien si ce n'est garder? Il ne transmet rien, n'offre aucune médiation: il empêche ou permet le passage mais ne le façonne pas. La mort de l'homme devant la porte ouverte souligne la parfaite imperméabilité de la loi et l'absurde de la promesse de son accessibilité à tous et toujours. Imperméabilité confinant à la forteresse par suppression de toute instance pouvant opérer médiation: pas l'ombre d'un juge, pas une seule référence à la justice. Kafka pose une loi absolue au sens génuine du terme: séparée de, coupée de; une loi qu'aucune finalité n'anime, une loi hiératique.

Succombant au premier élan, on trouvera même quelque réconfort à conclure à l'arbitraire du droit, mieux au gouvernement des juges, se remémorant les magistrats d'Ancien Régime qui se plaisaient, affirme-t-on, à parer de la plus noble équité velléités et autres prévarications. Malheureusement pour la tranquillité de nos âmes, il est à craindre que Kafka se veuille plus cruel, dénonçant nos temps bien davantage que récriminant contre le passé.

Violence symbolique de la loi - de cette loi, de quelle loi? - sans nul doute. Parce que la violence est encore un lien - comme l'atteste l'existence multiséculaire d'un droit de la guerre - et recèle donc quelque commensurabilité, nous proposerons, corroborant et noircissant l'hypothèse interprétative du «dérègle-
\end{abstract}

Au sens large de fabula: une séquence d'actions (U. Eco, De Superman au Surhomme, Grasset, Paris, 1993, 14). 
ment de la fonction symbolique» avancée par F. Ost ${ }^{2}$, la clef de la «panne symbolique».

Nous ne saurions toutefois user de cette clef à la manière du critique littéraire: la science nous en manque. Aussi considérons-nous la fabula de Kafka davantage «pré-texte» que texte, voire simple prétexte à une réflexion de philosophie contemporaine du droit autour de la possible construction de la commensurabilité entre loi et cas d'une part - problème classique de théorie du droit -, entre individu et collectif (représenté par la Loi) d'autre part - perspective de philosophie politique et plus radicalement point de vue ontologique.

Dès lors ne nous référerons-nous pas à la vie de Kafka, à la signification de cette nouvelle dans son œuvre, moins encore prétendrons dans la perspective herméneutique d'un Schleiermacher comprendre ce texte mieux que son auteur.

\section{TITRE 1. DE L'ABSENCE DE MÉDIATION À LA «PANNE SYMBOLIQUE»}

\section{Chapitre 1. L'incarnation de l'absurde}

Comment ne pas ressentir de l'absurde à la lecture de Devant la loi?

Pour conjurer l'aspiration du vide qui en résulte, l'homme tente d'en rendre raison, tâche éminemment délicate puisqu'elle ne peut être conduite que sous la menace permanente d' «autophagie »: l'absurde «rationalisé» est-il encore l'absurde?

On peut néanmoins tenter de présenter quelques instances de l'absurde.

Celle de l'inaccessibilité de la loi, explicite dans le texte de la fabula, ne retiendra guère notre attention comme juriste-citoyen d'un Etat de droit, au sein duquel la garantie d'un procès, plus d'un procès équitable, est très largement effective. La technique juridique actuelle fournit nombre de médiations, procédures de toutes sortes et de tous niveaux, multiplie les possibilités de médiations extra-judiciaires et d'arbitrage. Autant de voies vers la justice et de voix de la justice.

Devant la loi donne à envisager une autre instance de l'absurde à l'actualité plus évidente: un transport de sens qui ne s'effectue pas.

Nous avons déjà mentionné l'absence de pourvoyeur, de vecteur au moins, de la médiation, de passeur. Dans le domaine du droit, pareille absence se dénomme précisément «déni de justice »: un juge tarde gravement à rendre justice ou pis, le cas est ignoré, nié.

Il semblerait qu'ici, en effet, le cas soit connu et même une entrée possible identifiée; et pourtant l'homme ne rentrera pas dans la loi, la justice ne passera pas, faute de sens.

Mais quel contenu ou quel sens aurait-elle été capable de proposer, voire d'imposer? Où situer la «panne de sens »? Nulle part craignons-nous car de contenu, de «juste», il n'est fait aucune mention, pas plus que n'est formulée une prétention de quelque précision, nécessaire pourtant à l'évaluation de la pertinence d'une demande de justice.

\footnotetext{
2 F. Ost, Raconter la loi, Odile Jacob, Paris, 2004, 340 ss.
} 
Qu'importe, en effet, de soumettre une telle demande d' une certaine justice (la justice pour ce cas-ci) à un gardien? Il n'a pas pour fonction de juger mais de menacer, d'imposer le respect.

En lui s'incarne l'un des adages les plus brutaux du droit, au sens littéral «insensé » et donc absurde: la loi doit être obéie parce qu'elle est la loi. Moins un sens non-partagé, ou mal transporté, en d'autres termes un sens «raté », que l'absence de tout sens.

Autre formulation, tout aussi monolithique ou monologique que la précédente: l'ordre pour l'ordre. En celle-ci l'absurde dévoile l'un de ses ressorts: le moyen - l'ordre - devient explicitement la fin, sa propre fin - «pour l'ordre »; il absorbe la fin, consacre la perte du telos (de la téléologie), de la causalité finale, emblématique de la modernité verrons-nous plus en détail. L'absorption de la fin dans le moyen traduit dans les termes de l'ingénierie - la science des moyens précisément - le mythe moderne de l'auto-fondation, ou encore l'absence d'une altérité constitutive de soi et de la cité à la manière de l'animal politique (zoon politikon) des Anciens.

L'auto-fondation des Modernes couvre tout le champ de la praxis, de l'individu auto-fondé dans le solipsisme que le cogito cartésien place au principe de la métaphysique et de la science moderne, jusque sur le plan politique auto-fondé dans le contrat social, auto-fondation d'autant mieux réussie que le contrat social est pur de toute réalisation historique. Sous ces traits, le philosophe reconnaîtra la grande tradition, toujours vivace, du néoplatonisme ou des métaphysiques de l'Un dont découlent toutes les hypostases sans jamais l'épuiser.

Pour preuve de la brutalité de l'absurde soulignée, brutalité de la stricte juxtaposition, de l'absence de médiation, relevons qu'aucune obligation n'est formulée dans la loi de la fabula: ce serait là trop offrir, le possible d'un lien, le fameux vinculum juris des romains qui sous-tend toute obligation sur le modèle de la chaîne avec laquelle on attachait les esclaves ou les prisonniers (vinculum). La juxtaposition semble corroborée par l'attitude du gardien qui est de pure commination comme le relève $\mathrm{F}$. Ost ${ }^{3}$. On comprend à ce titre qu'un simple gardien suffit à la tâche.

Où l'on commence d'entrevoir que la juxtaposition équivaut à l'absence de respect, de considération. On se souviendra de l'importance première de la consideration dans l'application du droit anglais. Poli sans doute, le gardien ne témoigne aucun respect à l'endroit de l'homme de la campagne, à l'instar des «questions posées avec indifférence à la manière des grands seigneurs » (Kafka), «grands» et «seigneurs» pour asseoir dans un désarroi toujours plus grand l'incommensurabilité des positions, des pôles, ici l'homme, là la loi et son gardien. Désarroi augmenté encore par des indices d'ouverture privés d'effet: pseudo-dialogue («dia-logos », la parole, la raison qui va de l'un à l'autre, entre-deux) ici, là porte ouverte.

En d'autres termes, une cause ne parvient pas à devenir chose, res, réelle à prendre pied dans la réalité. Car là encore il faudrait une médiation, pour le moins

3 Ost, op. cit., 386.

${ }^{4}$ Rappelant que causa signifie aussi bien l'objet du procès, ce qui est en conflit que la chose, une res, un élément de la réalité et, évidemment, dans un sens scientifique, la cause au sens de l'antécédent de l'effet. 
celle transmuant le «fait» en «fait juridique», à savoir le prendre en considération, le revêtir de quelque pertinence pour le droit. La stricte juxtaposition soigneusement exprimée semble suggérer davantage l'absence de sens, et même de tout sens possible - par absence de lien, de relation - qu'un sens qu'on ne parvient pas à transmettre: faux dialogue homme (de la campagne)-loi, hommegardien et, plus abstraitement, cas-loi.

Face à pareille situation, l'esprit aura tôt fait de conclure à l'arbitraire, caricaturé à l'extrême dans le recours à la prière des puces pour changer l'humeur du gardien. L'esprit profane semble au reste bien préparé à tel «constat» tant la méfiance à l'égard de la justice comme œuvre d'un juge est de tous horizons nourrie. Nous avons toutefois relevé l'absence de juge, la seule personne ayant quelque lien avec la loi étant, dans la fabula, le gardien, officiel ne revêtant aucune fonction de médiation, de passeur: son simple office désigne ce qu'il garde - la loi - comme forteresse, rendant immédiatement improbable l'élaboration d'une commensurabilité indispensable à l'assomption du cas par et «dans» la loi.

La situation kafkaïenne illustre sans doute, prima facie, un déni de justice: la justice y est bel et bien niée, jusque dans ses conditions de possibilité pour reprendre un langage kantien; elle ne parvient pas même à «passer».

Aussi prêterons-nous au malaise provoqué par Devant la loi une cause plus radicale que celle d'une justice qui aurait pu être rendue: l'incommensurabilité du cas et de la loi est proprement insurmontable, et pour une cause remontant jusqu'aux origines du politique (au sens de social): le symbole dont le dysfonctionnement est mis en scène.

\section{Chapitre 2. Derrière l'absurde, la panne symbolique}

Nous avons suggéré une sombre hypothèse de lecture, la panne symbolique, la reliant à un «constat» de lecture, la stricte juxtaposition des protagonistes.

Cette hypothèse prend source dans la notion originelle de symbole. Le sumbolon vient du verbe sumballein «jeter ensemble», «joindre, réunir » «mettre en contact $»^{5}$. Il implique deux pôles et, entre eux, une relation («ensemble», «en contact»). Si Devant la loi exhibe bien des pôles - le campagnard et le gardien -, aucune relation en revanche ne prend chair dans la fabula, laquelle se contente de les strictement juxtaposer, à savoir les poser l'un à côté de l'autre et donc pas « ensemble »: ils ne forment rien entre eux, il ne (se) passe rien non plus entre eux.

Le tragique de la situation devient l'absurde d'un symbole privé de son ressort. On doit ajouter, redoublant de la sorte la charge contre la «Loi », qu'il n'est guère d'institution plus symbolique que la loi précisément. L'institutionnel se trouve, en effet, au cœur du symbole: «De par l'origine première du terme (le tesson [symbolon] de poterie servant de signe de reconnaissance aux partenaires d'un pacte), le symbole appartient à l'ordre de la communication: il est le lieu où un monde naît comme monde commun, intersubjectif $»^{\circ}$.

${ }^{5}$ Dictionnaire historique de la langue française, Dictionnaires LE ROBERT, Paris, 1992, entrée «symbole».

${ }^{6}$ Les Notions philosophiques, Encyclopédie philosophique universelle, 2 tomes, PUF, Paris, 1990 , entrée «symbole» $[\mathrm{rel}]$. 
La béance qu'ouvre Devant la loi s'éprouve vertigineuse. Avec la panne symbolique, on perd toute possibilité de reconnaissance, perte de l'altérité constructive, constitutive, déjà esquissée dans le manque de respect, lequel renvoie étymologiquement au manque de «ré-flexion» dans le miroir soit l'absence de reconnaissance et donc la panne du symbolon. La «com-munication» disparaît également dans le gouffre, dans lequel s'abîme corrélativement l' «intersubjectivité ». Et pourquoi les deux tessons de la relation (de justice ici) ne sontils pas adéquats l'un à l'autre? Parce que la relation de justice (pour «loi»), ou plus largement d'autorité (pour «Loi») étant toujours à faire ou à dire - comme l'indique explicitement le mot «juris-dictio»-, relation dynamique, à contextualiser, on ne peut y parvenir à partir d'une position statique non relationnelle comme la juxtaposition, en s'arque boutant sur les seuls pôles, seuls au sens fort du solipsisme moderne.

Le modèle statique ou polaire a perdu toute pertinence en sciences dures. Abandonné le mythe de l'observateur neutre; la nature ne répond que provoquée et donc préparée pour l'expérimentation, médiatisée par des instruments, des choix d'échelle, etc. A la vérité absolue et ses faits bruts se sont substitués la pertinence et ses faits «aménagés », la science devenant un dialogue rationnel et expérimental entre le Sujet et l'Objet, qui «renvoie à deux dimensions constitutives des rapports homme-nature: comprendre et modifier. L'expérimentation ne suppose pas la seule observation fidèle des faits tels qu'ils se présentent, ni la seule quête des connexions empiriques entre phénomènes. L'expérimentation exige une interaction entre théorie et manipulation pratique, qui implique une véritable stratégie. Un processus naturel se trouve arraisonné comme clef possible d'une hypothèse théorique; et c'est en tant que tel qu'il est alors préparé, purifié, avant d'être interrogé dans le langage de cette théorie.» ${ }^{7}$ La stratégie suppose évidemment une fin, un but ou cause finale. K. R. Popper parlait d'un «horizon des attentes » en ce sens qu' «il n'existe pas d'observation impartiale. Toute observation est une activité qui a un but (trouver, ou contrôler, une régularité qui est, pour le moins, vaguement envisagée); une activité dirigée par des problèmes, et par le contexte d'attentes («l'horizon des attentes» [...]). L'expérience passive n'existe pas $[\ldots] .{ }^{8}$

Pourtant la métaphysique moderne demeure très largement polaire ainsi que le droit et pour la même raison principielle: la perte de la causalité finale ou l'homogénéisation du monde par réduction des phénomènes à des jeux de forces, physiques ou sociales suivant les domaines, règne de la causalité efficiente, de la potestas, qui ne connaît que le langage de la puissance, du pouvoir. Devant la loi expose des gardiens toujours plus puissants, à la puissance proprement incommensurable même, insupportable (dès le quatrième). Le Titre 2 interrogera et l'ontologie et le droit sur la perte de la causalité finale, laquelle équivaut à une perte de la relation en tant qu'une causalité mécanique (à l'exemple de la causalité efficiente) expulse théoriquement tout choix au profit du calcul.

7 I. Prigogine/I. Stengers, La nouvelle alliance, Gallimard, Paris, 1990, 32.

8 K. R. Popper, La quête inachevée, Presses Pocket, Paris, 1989 (1 $1^{\text {èr }}$ édition française CalmannLévy, 1981), 68. 
Kafka poussera la cruauté jusqu'à mettre en présence les deux hommes mais en refuant toute «inter-subjectivité », cet «entre» les sujets: une simple coprésence, marque d'un monde polaire. Les philosophies polaires qui lui répondent surdéterminent le pôle par rapport à la relation, laquelle est censée n'être que la résultante de ces pôles, dans la mesure où ils ne se trouvent aucunement constitués par elle. Ils sont les sujets premiers dont elle découle.

Dès lors la relation simplement «accidit» dit le latin, elle s'ajoute comme un accident $\left(\right.$ au sens philosophique ${ }^{10}$ ) à quelque chose qui est déjà là et complet en son essence et dont le passage à l'acte - la concrétisation dans le monde des existants - ne change rien à sa nature profonde. Manière de quintessence du paradigme analytique au sens kantien du mot $^{11}$ (et non anglo-saxon). Le «passage à l'acte» - formule courante sans doute mais traduisant au plus près le dynamisme de la pensée aristotélicienne construit notamment autour du couple puissance/acte -, n'est-ce point ce qui fait défaut, défaille tout au long de la fabula? La fabula s'interrompt précisément au moment où le passage à l'acte aurait pu se «réaliser» au bénéfice de l'homme de la campagne, quand «la» porte est identifiée comme «sa » porte: la cause est sur le point - peut-être - de devenir chose et s'inscrire enfin dans le réel, la promesse se réaliser.

Les théoriciens contemporains du droit reconnaîtront dans Devant la loi le parangon de la loi hiératique parce qu'essentialisée, réifiée: une loi-masse, une loi-forteresse, animée d'aucun mouvement c'est-à-dire non pénétrée de la destination première du droit qui est l'application, précisément le passage à l'acte. Le couple puissance/acte traverse la pensée d'Aristote en tant qu'elle vise principiellement à appréhender le mouvement, le devenir soit une position non essentialiste. La primauté de la perspective de l'acte, aussi bien cognitive qu'ontologique, permet d'entendre littéralement que l'existence (point de vue de l'acte) «par-fait »

\footnotetext{
9 On sait combien seront lourds les moyens théoriques mis en œuvre par la philosophie politique moderne pour justifier le social, ou politique ou institutionnel à partir de purs pôles: le monstre Léviathan (Hobbes), la volonté générale autorisée à mettre à mort l'individu-citoyen récalcitrant après passation du contrat social (Rousseau), des principes contrefactuels (de Rawls à Habermas).

${ }^{10}$ «Ce terme s'oppose à essence ou substance pour désigner ce qui existe non en soi-même mais en une autre chose et révèle «les manières particulières d'exister de la substance» (Kant, Critique de la raison pure, Analyt. II, 1re analogie de l'expérience)»(Les Notions Philosophiques, op. cit., 18).

${ }^{11}$ «Dans tous les jugements, où est pensé le rapport d' un sujet au prédicat [...], ce rapport est possible de deux façons. Ou bien le prédicat B appartient au sujet A comme quelque chose qui est contenu (de manière cachée) dans ce concept $A$; ou bien $B$ est entièrement hors $d u$ concept $A$, quoique en connexion avec lui. Dans le premier cas je nomme le jugement analytique, dans l' autre synthétique. Des jugements analytiques (affirmatifs) sont donc ceux dans lesquels la connexion du prédicat avec le sujet est pensée par identité, tandis que ceux dans lesquels cette connexion est pensée sans identité doivent s'appeler justement synthétiques. On pourrait encore appeler les premiers jugements explicatifs, les seconds jugements extensifs, car ceux-là n'ajoutent rien, par le prédicat, au concept du sujet, mais le décomposent seulement par analyse en ses concepts partiels, qui étaient déjà pensés (quoique confusément) en lui : tandis qu'au contraire les seconds ajoutent au concept du sujet un prédicat qui n'était pas du tout pensé dans le sujet, et qu'aucune analyse de celui-ci n'aurait pu en tirer.» E. Kant, Critique de la raison pure, «Introduction», in Euvres philosophiques I, Gallimard, La Pléiade, Paris, 1980,765 .
} 
l'essence (point de vue de la puissance) ${ }^{12}$ c'est-à-dire la fait être de part en part, l'achève.

L'acte s'éprouve intimement lié à la fonction, à la finalité (cause finale) comme le met didactiquement en exergue Aristote avec l'épisode de la main coupée. La main ne peut réaliser ses potentialités, exercer sa fonction naturelle, qu'au sein d'un corps vivant, sans lequel elle sera inerte, inanimée, de nul usage. C'est pourquoi la main coupée, n'étant plus partie d'un corps vivant animé c'està-dire apte à passer à l'acte sous l'action de l'âme (anima), ne se dit main que par homonymie, selon un pur jeu de langage qui ne correspond à plus à aucune réalité pratique. Dans ce sens pragmatique (pragma est la chose concrète en grec), le tout précède les parties.

Aussi peut-on caractériser la position essentialiste par la perte de la causalité finale, la perte de la destination première de la loi n'étant qu'exemplaire de cette perte de la fin. Peut-être devrait-on parler de panne de la finalité, au reste bien connue des juristes dont la théorie traditionnelle de l'interprétation relègue au dernier rang la méthode dite téléologique. Ou encore, plus gravement, s'agit-il d'une panne du relationnel en général, laquelle condamne les protagonistes à des positions simplement juxtaposées et prive d' «opération» le symbole institué - ici la loi, institution éminemment symbolique -, le rend hiératique?

La panne symbolique recevrait de la sorte une portée encore plus vaste et plus perturbante. Dans la lecture philosophique du symbolique, celle de Ricœur par exemple (De l'interprétation et La métaphore vive), le symbole intervient - littéralement «vient entre » donc médiatise - doublement dans l'élaboration du sens: il vaut principe, arche, découvrant sa nature matricielle et indique simultanément la fin, le but, telos, opérant attraction et condensation de sens.

Soulignons la simultanéité de l'arche et du telos comme deux instances distinctes mais intrinsèquement corrélatives du même phénomène suivant la logique aristotélicienne du couple puissance/acte: deux modes corrélatifs du réel suivant le modèle du passage à l'acte «par-faisant », faisant «être » de part en part, achevant la puissance ou potentia dans l'acte, lequel la réalise au sens propre, la rend réelle.

La Loi comme promesse de justice - «une glorieuse lueur» (Kafka) - ne parvient pas à passer à l'acte, elle ne concrétise pas sa potentia: la cause ne peut devenir chose, prendre place dans le réel (res donnera «chose» et «réalité»). Comment pourrait-il en aller autrement dans Devant la loi: si le symbole est porteur d'arche et de telos, la panne symbolique prive alors les protagonistes et d'un principe et d'un but: rien à l'origine, rien à la fin? L'absurde s'éprouve fondamental et intégral, et nullement accidentel.

C'est donc moins tel sens de la loi (telle interprétation du texte légal) qui ne passe pas que la loi elle-même, l'institution «Loi», emblématique de tout «institué», qui ne parvient pas à faire lien, à relier, à médiatiser et donc à faire sens.

Que la panne symbolique soit une panne de l'institution au sens de l'action d'instituer (et non son résultat comme tel) et donc de l'acte (du couple puis-

${ }^{12}$ Sur ces points et leur assomption dans la perspective englobante d'un aristotélisme dynamique, voir A. Papaux, Essai philosophique sur la qualification juridique: de la subsomption à l'abduction, Paris-Bruxelles-Zurich, LGDJ/Bruylant/Schulthess, 2003, 171 ss. 
sance/acte) - ou de manière redondante du passage à l'acte - consone avec la structure même du symbole telle que l'analyse une certaine sémiotique, celle de C. S. Peirce et de U. Eco en l'occurrence.

On distingue alors le symbole de l'icône et de l'indice suivant le type de relation qu'ils postulent entre leurs pôles constitutifs, «l'icône, opérant par similitude de fait entre signifiant et signifié; l'indice, par contiguïté de fait; le symbole, par connexion apprise formant règle $»^{13}$, ces distinctions n'étant jamais que relatives. Icône et indice sont des types de signes présentant un certain lien «naturel», non strictement conventionnel, entre leurs pôles. Ils recèlent donc une certaine motivation dans le langage de Eco. Au contraire, le symbole ne se fonde sur aucune motivation (similitude ou contiguïté «de fait») pour opérer liaison: il est le signe dont l'aspect conventionnel est le plus marqué et qui laisse donc le locuteur dans la dépendance la plus grande face au collectif, à l'institué et à l'institution.

Le symbole en cela exprime la forme la plus aboutie de la juxtaposition, à laquelle correspond dans la langue philosophique le qualificatif d' "absolu» à savoir séparé de, coupé de. Tel sera au Titre 2 le sort de l'individu moderne, pur pôle, solipsisme ontologique, d'une part, de la loi juridique moderne coupée de ses applications, ignorant (en théorie seulement) contexte et juge, d'autre part.

\section{TITRE 2. PHILOSOPHIE POLAIRE OU MÉTAPHYSIQUE DE LA JUXTAPOSITION}

\section{Chapitre 1. Ontologie polaire, perte de la finalité et absence d'altérité constitutive}

Le symbole condense magnifiquement la figure de l'altérité constitutive de la relation et le potentiel d'institutionnel qu'elle porte, en particulier dans le schème de la reconnaissance, ressort même du symbole et dont les deux tessons sont comme les preuves matérielles.

Or l'ontologie moderne se caractérise par l'absence voire la négation de toute altérité entendue au sens d'une altérité constitutive, non de toute altérité comme telle. Descartes peut ici produire maints titres de paternité. On se souvient du solipsisme que l'on dit ouvrir la modernité, le fameux «je pense donc je suis » (cogito ergo sum). Forme éminente du monologue: aucune parole ou raison ne passe - comme dans le «dia-logue»-, le «je»se contentant de «ré-fléchir» le «je » dans un dédoublement qui semble créer de l'épaisseur, de l' «entre » mais qui n'est jamais que l'expression renouvelée de l'homogénéité, pensée se développant dans le même genre («homo-gène») à savoir sans altérité, comme dans le monologue où la parole formulée ne s'adresse à personne, sans «autre» pour la recevoir.

Cette homogénéité est au reste si marquée que Descartes ne saura comment sortir du «je» pour gagner quelque altérité. Même une hypothèse aussi forte que celle du malin génie échoue à briser le monologue ou l'enfermement dans la res

\footnotetext{
${ }^{13}$ Les Notions Philosophiques, entrée «symbole» [rel].
} 
cogitans, la chose pensante $^{14:}$ les sens nous trompent répétera Descartes, indiquant que le monde extramental lui-même ne parvient pas à imposer quelque altérité pertinente. Comment, dès lors, sortir du pôle «je», du solipsisme? Descartes avance une solution si forte et si dispendieuse que le rationalisme ne pourra l'assumer sans une grande perte de crédit: la garantie divine. Assuré «rationnellement» (via les preuves de l'existence de Dieu) de l'existence et de la bonté de Dieu, il peut croire à la correspondance entre ses idées sur le monde extramental et les réalités de ce monde.

Cette médiation divine, pas plus que celle de la glande pinéale, ne convaincront qu'est de la sorte surmonté le dualisme entre la chose pensante et la chose étendue. En bref, l'élaboration de la commensurabilité demeure en souffrance d'explication.

Il n'empêche que la res extensa, la chose étendue présente toutes les qualités pour recevoir un traitement mathématique, bien préparé au reste par Galilée et sa fameuse métaphore du Grand Livre de la Nature - écrit dans le langage de la mathématique affirmera précisément le mathématicien «italien», laquelle fut rendue possible par le contexte religieux de l'époque souligne le physicien J.-M. Lévy-Leblond ${ }^{15}$.

L'oubli de la dimension religieuse de la science moderne au profit d'une opposition absolue entre les deux domaines signe le projet moderne, vœu pie à suivre B. Latour: à la philosophie de la pureté, des pôles, en particulier pôle-Sujet et pôle-Objet, parfaitement distincts, il oppose une pensée des hybrides, des mixtes de Sujet et d'Objet, point de départ éminemment pragmatique d'une connaissance s'induisant (s'abduisant en vérité) d'incessantes rétroactions des instances ou entités les unes sur les autres. L'entreprise moderne reste au contraire profondément marquée par une propension aux dualismes, aux modes binaires, à tout le moins polaires, de penser. La juxtaposition des éléments obligera souvent à des coups de force pour surmonter cette manière de nominalisme: existence de jugements a priori et pourtant synthétique (Kant), idées innées, doctrines du contrat social, conditions contra-factuelles, etc.

Quoi qu'il en soit, découle de cette mathématisation autant qu'elle la cause en rétroactions successives une homogénéisation du réel. Le réel s'éprouve «naturellement» univoque ensuite de la réduction de la pluralité des causes aristotéliciennes à une unique cause, la causalité efficiente en l'occurrence, la seule qui ne soit ni triviale - comme la causalité matérielle - ni mystérieuse, magique voire mystique - comme les causalités formelle et finale. La causalité efficiente se

${ }^{14}$ Référence aux deux essences que Descartes «découvre» au fil de ses Méditations métaphysiques : chose pensante d'une part, chose étendue (res extensa) d'autre part. Ce dualisme, qui marquera profondément la philosophie moderne, s'avère insurmontable pour toute philosophie polaire, à l'enseigne du cartésianisme.

15 J.-M. Lévy-Leblond, «La science est-elle universelle?», in Alliage, $\mathrm{N}^{\circ}$ 55-56, Editions du Seuil (diffusion), Paris, 111. Religion et science n'apparaissent pas en si radicale opposition que ce que feront accroire Les Lumières. Leurs relations sont infiniment plus complexes, à l'exemple du parcours de Galilée longtemps soutenu par le Pape (voir G. Minois, Galilée, PUF, QSJ, Paris, 2000 ou encore K. R. Popper, dans Conjectures et réfutations, Payot, Paris, 1985, 150 ss. montrant que l'opposition de l'Eglise n'est pas directement scientifique mais politique: pour des raisons de pouvoir donc de puissance, potestas. 
traduira rapidement en physique moderne par l'idée de force, vivement promue par Newton - là encore non sans arrières pensées considérées aujourd'hui comme « irrationnelles », à savoir l'alchimie pour le fondateur de la Royal Society.

La traduction de la causalité efficiente en force ou puissance c'est-à-dire potestas rencontrera parfaitement l'aspiration des humanités au statut de science, fascination qui les conduira souvent au scientisme.

Un premier rapprochement s'opérerait aisément sur base de potestas : la praxis n'était-elle pas tissée de forces sociales en perpétuel conflit, parfois en équilibre? Le droit n'était-il point en train d'opérer sa migration d'une version (plutôt) objective vers une version (plutôt) subjective par le ministère des «droits subjectifs", droits rattachés à la personne comme partie de l'humanité ou plus radicalement à l'individu sous la poussée du nominalisme? Le droit se conçoit désormais principalement comme le pouvoir (potestas) d' un individu, une sphère de compétences se développant à partir de chaque sujet (subjectum), conception largement passée dans la doxa (l'opinion publique) sous la forme canonique du «j' ai le droit de...». Mais si le droit est principiellement affaire de pouvoir, le plus (par où s'exprime bien une homogénéité) puissant l'emporte comme le relèvera un Hobbes prophétique définissant, dans le De Corpore (Chap.6, §7), la loi comme le «commandement de celui qui peut contraindre» ensuite de la «volonté des hommes qui ont établi par souci de paix un tel pouvoir», sachant que «si les instincts et les passions des hommes ne sont pas contenus par quelque pouvoir, ils se combattront entre eux...».

En sortira la liberté des Modernes, aussi pénétrée de volonté que de potestas, deux notions corrélatives, synthétisée par Burdeau comme «la faculté que tout homme porte en lui d'agir selon sa détermination propre sans avoir à subir d'autres contraintes que celles qui sont nécessaires à la liberté des autres $»^{16}$. Aucune trace de causalité finale, absence complète d'inclination «naturelle», à vivre en société par exemple (l'homme, animal politique des Anciens).

En d'autres termes, autrui ne s'éprouve jamais intrinsèquement constitutif de l'identité de l'homme qui demeure, théoriquement, un strict individu, dans une veine nominaliste. Le pouvoir «in-fini» de l'individu, au double sens de pur de toute fin naturelle et d'illimité, fut consacré en droit sous l'espèce du droit de propriété conçu absolu (c'est-à-dire séparé de, coupé de) comme à l'article 544 du Code Napoléon: «droit de jouir et disposer des choses de la manière la plus absolue [...]».

Dans ce contexte, la présence de l'autre apparaît bien accidentelle ( $c f$. solipsisme). C'est donc d'un accident que vient l'éventuelle limitation d'un pouvoir en soi illimité si caractéristique des Modernes: «Le monde moderne est né sur les décombres des systèmes symboliques traditionnels, en qui il n'a su voir que de l'irrationnel et de l'arbitraire. Dans son entreprise de démystification, il n'a pas compris que ces systèmes impliquaient que des limites soient fixées à la condition humaine, tout en leur donnant sens. En remplaçant le sacré par la raison et la science, il a perdu tout sens des limites et, par là même, c'est le sens qu'il a sacrifié.» ${ }^{17}$ On comprend que la relation, ici l'altérité, ne saurait être constitutive

${ }^{16}$ G. Burdeau, Le libéralisme, Paris, 1979, 40.

17 J.-P. Dupuy, Pour un catastrophisme éclairé, Seuil, Paris, 2002, 57. On lira dans le même sens, avec le thème classique de 1'hybris, A. Finkielkraut, Nous autres, modernes, ellipses, Paris 2005, en particulier 185 ss. 
des pôles (en particulier les individus) car elle viendrait limiter intrinsèquement leur pouvoir, les déterminer. L' «auto-nomie», autre caractéristique moderne, illustre parfaitement bien la juxtaposition. Elle ressortit elle aussi à la liberté des Modernes suivant la synthèse de Burdeau.

La définition de Burdeau, au reste, traduit le tour d'esprit le plus classique des doctrines du contrat social, dans lequel se reconnaît une transposition de problèmes physiques d'équilibre entre entités individuelles (parallèlement à l'individu atome sociétal), entre «corps» comme celui des trois billes au fond du bol et dont l'équilibre résulte d'un pur jeu mécanique des forces qui s'«entre-équilibrent $»$, solution constatable quoique mystérieuse en son fond puisque le problème des trois corps - pour ne parler que de notre soleil, de la lune et de la terre - n'est toujours pas résolu.

Un second rapprochement, plus manifeste encore, fut porté par maints juristes, en particulier dans l'Ecole moderne du droit naturel, lesquels firent accroire que les notions de lois étaient identiques en physique et en droit, sous l'angle de la régularité (regula en latin, la règle [de droit notamment]). Fort de cette ambiguïté entretenue, et déterminés à fonder une Rechtswissenschaft, d'aucuns poussèrent jusqu'à adopter la démarche mathématicienne du more geometrico, persuadés en particulier que le droit codifié (les codes) s'appliquerait par déduction des principes généraux aux cas singuliers.

Pufendorf exprimera sans ambages cet emprunt, ce calque de la science mathématique: «Il y a à mon avis deux voies que l'on peut suivre si l'on entend donner une forme scientifique à une discipline. L'une est celle que suivent les mathématiciens qui aiment à développer à partir de quelques principes peu nombreux une masse de propositions. L'autre est celle que prennent ceux qui ont à cœur l'étude des choses de la nature quand ils infèrent de l'observation et de l'accumulation d'une multiplicité de choses singulières une loi générale. Beaucoup m'ont engagé à suivre cette dernière voie en cette matière. Il faudrait en vérité rassembler alors tout ce que les différents peuples considèrent comme Droit et ce sur quoi tous et chacun tombent d'accord. En réalité, cette voie est incertaine, infinie et presque impraticable... car je crois qu'il n'y a pas une prescription du Droit naturel que ne contredisent les mœurs ouvertement admises de n'importe quel peuple (...) Après avoir ainsi rejeté cette méthode, je pense qu'on doit plutôt suivre ici les mathématiciens, c'est-à-dire qu'il faut établir un principe immuable que personne de sensé ne peut mettre en doute et dont alors tout ce qui est de Droit naturel et durable puisse procéder.» ${ }^{18}$

Le droit comme pouvoir ou puissance, le droit réduit à un code, la démarche verticale et descendante à partir de lois a priori, des applications réduites à de simples accidents, autant de figures logiques au travers desquelles s'esquisse un droit-commandement, un jus potestas, par contraste avec un droit-relation, un droit-mesure: «la règle devient ce qu'il faut faire ou ne pas faire pour être dans la norme, c'est-à-dire un impératif assorti des sanctions ${ }^{19}$, esprit bien différent du

${ }^{18}$ Pufendorf cité par A. Dufour, Droits de l'homme. Droit naturel et histoire, PUF, Paris 1991, 107-108.

19 A. Sériaux, Le droit naturel, PUF, QSJ, Paris, 1993, 76-77. Pour de plus amples développements, voir A. Papaux, op. cit., 119 ss. en particulier 123 ss. 
classique suum cuique tribuere (rendre à chacun la part juste, la part «qui lui revient») issu du droit comme ars boni et aequi, art du bon et de l'équitable. La notion d'ordre change corrélativement de signification: «ordre devient synonyme de commandement alors que chez les Anciens il avait le sens d'un agencement correct des choses ou des personnes entre elles ${ }^{20}$.

Le droit-puissance (jus potestas) ayant réduit la praxis à n'être que la résultante, à la manière du parallélogramme des forces, du jeu des forces sociales, de l'équilibre des pouvoirs et des contrepouvoirs, il s'offre, par postulation, un réel homogène. Entre les multiples pôles les différences existent mais se réduisent à une considération de quantité comme le souligne Hobbes: le plus puissant commande, le gouverné obéit c'est-à-dire réduit sa sphère de pouvoirs, ses «droits de».

Comment dans pareil cadre concevoir la rencontre entre la loi générale et abstraite et le cas singulier et concret, comment penser le saut qualitatif qui les sépare et que le juriste doit franchir, obligation lui étant faite de résoudre les litiges à lui soumis sous peine de déni de justice?

Certes y a-t-il encore un peu de relation entre les entités prises dans une confrontation quantitative de puissance. Les juristes l'affirment à propos de la relation la plus tenue entre Etats existants mais relation encore, à savoir la guerre et ses deux principaux corpus de droits: jus ad bellum et jus in bello. Le peu de respect pratique dont bénéficie ce dernier montre que l'on est au plus bas degré de la relation, à l'extrême limite de sa négation c'est-à-dire la juxtaposition, forme discrète de la non-considération voire de la négation de l'autre, à tout le moins de l'autre comme altérité constitutive de sa propre identité dans le type de relation considérée (ici le droit international, là la justice).

Cette logique relationnelle de guerre et paix est d'analyse très difficile, et évidemment trop ramassée ici, en tant notamment qu'elle est recouverte d'un voile mythologique, entendu que «la paix par le droit a toujours été un mythe ${ }^{21}$. Le philosophe Alain souligne dans un Propos au titre inverse, «Le droit par la paix », que la paix et, par conséquent, une certaine relation de reconnaissance entre les parties, doit précéder le droit pour que celui-ci puisse advenir: «Au contraire, c'est le droit qui sera par la paix, attendu que l'ordre du droit suppose une déclaration préalable de paix, avant l'arbitrage, pendant l'arbitrage et après l'arbitrage, et que l'on soit content ou non. ${ }^{22}$

$\mathrm{Si}$ la relation de reconnaissance est constitutive du droit, on rappellera qu'elle est aussi fondatrice du symbole, aussi bien sur le plan matériel des tessons qui épousent le contour l'un de l'autre que sur le plan intellectuel d'une analogie «ana-logos» - qui permet la «re-connaissance» entre les pôles donc leur «connaissance à nouveau» (d'où l'«ana-logie»), expression d'une relation qui les constitue comme pôles.

Devant la loi, privé de la relation, pis privé de la relation de reconnaissance, abandonné dans la juxtaposition des pôles, comment concevoir épistémologique-

${ }^{20}$ Sériaux, 77.

${ }^{21}$ R.-J. Dupuy, Cours Général, Recueil des Cours de l'Académie de droit international, Sijthoff, Leyde, $1979 \mathrm{IV}, 194$.

22 Alain, Propos I, La Pléiade, Gallimard, Paris, 1956, 484-485. 
ment la rencontre des deux instances de la relation légale a priori incommensurables, hétérogènes: la loi en tant que générale et abstraite et le cas en tant que singulier et concret?

\section{Chapitre 2. Métaphysique de la juxtaposition ou de l'impossible assomption du cas par la loi}

Adoptant la perspective de l'épistémologie juridique, nous nous contenterons de la seule question de l'assomption de l'idiosyncrasie par le général, problématique que Devant la loi installe dans une roborative «absurdité », roborative pour les juristes à tout le moins.

Si l'on s'en tient à la traduction française, la nouvelle s'intitule Devant la loi et non Devant le droit. La nuance sera déterminante, nous le verrons.

Cette absurdité signifie-t-elle que la loi, dans le moment même de son application, ne peut que se trahir en abandonnant sa généralité, voire en perdant son «sacré», se commettant avec le tout-venant (les gens de la campagne)? Dévoiement qui s'opérerait sans pour autant atteindre au singulier comme tel, à savoir l'idiosyncrasie: l'homme n'entre pas dans la loi.

A tout prendre, la loi ne devrait-elle pas demeurer dans une posture hiératique, celle que met en scène Kafka: la rencontre entre loi et cas ne se fait pas, la loi ne peut s'abaisser au particulier, à telle enseigne que ne figure dans la fabula aucune instance, aucun juge, aucun mode de la «juris-dictio», du «dire-le-droit». Simplement des gardiens dont la fonction est par définition anti-herméneutique, antipasseur: ils ouvrent ou ferment mais ne «trans-portent» pas, ne médiatisent rien.

Aussi, à la question de l'assomption de l'idiosyncrasie par le général répondrons-nous d'emblée, en tant que représentant de la Rechtswissenschaft, de la science juridique, par la négative.

Aristote n'avait-il pas relevé que du singulier il ne saurait y avoir science? A l'encontre de la prévention de maint esprit moderne, le Moyen Age ne fut guère thomasien $^{23}$ mais bien davantage augustinien (néplatonicien-chrétien-patristique) jusqu'à prendre le contre-pied de l'épistémologie aristotélicienne en affirmant que la connaissance intellectuelle du singulier comme tel est possible, de par la toute-puissance divine, à la manière de l'illuminatio augustinienne. Duns Scot atteindra même à l'idiosyncrasie, théoriquement à tout le moins, avec son concept d'haeccéité. Dans la même veine, on n'hésitera pas à affirmer la possibilité d'une connaissance vraie d'un objet qui n'existe pas. Où l'on perçoit que l'existence est un accident - les pragmata (les existants) ne constituant pas le point de départ de la pensée -, ouvrant alors la voie aux «expériences de pensée» des mondes possibles. Démarche intégralement idéaliste, éminemment si l'on considère que la source de pareille connaissance est Dieu, logique top-down, déductive en quelque sorte, que nous ne retiendrons pas $\mathrm{ici}^{24}$.

\footnotetext{
${ }^{23}$ On se souviendra de la condamnation de plus de 100 thèses de Thomas d'Aquin en 1277 par Etienne Tempier, évêque de Paris.

${ }^{24}$ La position scotiste nous semble au principe de la croyance positiviste ensuite de laquelle le raisonnement juridique s'opérant selon la forme (eidos - forma - idées - idéalisme) du syllogisme et la logique de la déduction permet d'atteindre la solution du cas à partir du seul texte de loi («cas » que l'on appelait jadis espèce, formule qui présentait l'avantage d'expliciter le déficit de singularité ou
} 
Au contraire l'affirmation aristotélicienne de l'impossibilité d'une science du singulier nous semble confirmée par Devant la loi en le chef de cet homme de la campagne, singulier certes, mais étrange singularité en tant qu'il ne porte point de nom propre. Serait-ce à dire qu'au moment même où l'on tente d'appréhender le particulier comme tel, il se dérobe et ne sera connaissable que sous l'empire de quelque généralité, elle-même fruit d'une certaine généralisation, minimale peutêtre pour l'espèce (infra note 24) mais déjà abstraite? On se trouve face à un particulier mais que l'on peut répéter au sens où se trouvera d'autres particuliers de la «même espèce »: le particulier ne se saisit que sous le mode de cette «répétition », de cette régularité (regula en latin, règle) et la loi s'enferme comme sur elle-même relativement à la particularité comme telle, à l'idiosyncrasie qu'elle manque.

Aristote le soulignait déjà il y a environ 2300 ans: la loi ne peut saisir que les cas les plus fréquents, elle s'avère nécessairement, intrinsèquement insuffisante: «la loi est toujours quelque chose de général, et [qu'] il y a des cas d'espèce pour lesquels il n'est pas possible de poser un énoncé général qui s'y applique avec rectitude. Dans les matières, donc, où on doit nécessairement se borner à des généralités et où il est impossible de le faire correctement, la loi ne prend en considération que les cas les plus fréquents [...]. La loi n'en est pas moins sans reproche, car la faute n'est pas à la loi, ni au législateur, mais tient à la nature des choses, puisque par leur essence même la matière des choses de l'ordre pratique revêt ce caractère d'irrégularité.» ${ }^{25}$ La regula ou régularité s'entend donc bien d'un «le plus fréquent». Les cas «marginaux», non-standards, ne sont pas assumés par la loi. Mais cette marge est depuis longtemps investie par le droit via le raisonnement par analogie notamment, droit qui, par là, se distingue nettement de la loi. La loi ne constitue que l'un des modes d'expression du droit, son mode écrit, quasi monopolistique dans la représentation occidentale actuelle du phénomène juridique.

La loi-regula laisse aussi de côté l'idiosyncrasie du cas, et si l'on peut dire «dans tous les cas» et non de façon simplement marginale. Et pourtant, si l'essence du droit est la justice, le jus ou juste, ne doit-il pas justement (en tous les sens que la langue française prête à cet adverbe) assumer l'idiosyncrasie? Répondre par l'affirmative emporte une sensible relativisation, de la science du droit. Mais qui veut maintenir la prétention à la scientificité du droit s'expose à en perdre la destination ultime: la justice du cas, rendre justice aux particuliers.

Quoi qu'il en soit, voilà le juriste précipité dans le désarroi, celui dont fait état le texte de synthèse du présent séminaire (supra P. Livet/G. Timsit).

Le désarroi des sciences sociales prend un tour singulier avec le droit. D'une part, historiquement, le droit consacre pour la qualification de son activité l'ensemble des solutions imaginables: d'origines religieuses, passant par l'ars aequi et boni, pour s'afficher aujourd'hui Rechtswissenschaft. D'autre part, matérielle-

d'idiosyncrasie de la démarche: partant du genre (général), on n'atteignait guère mieux qu'à l'espèce donc ce qui est commun à une série de particuliers). La «subsomption» ramasse l'ensemble de cette démarche en en masquant la contradiction logique que seule la toute-puissance de Dieu permettait de surmonter, référence de laquelle ne s'autoriserait que difficilement un juge contemporain. L'obligation de motiver sa décision avec de pareils arguments lui rendrait la tâche pour le moins difficile.

${ }_{25}$ Aristote, l'Ethique à Nicomaque, traduction Tricot, Vrin, Paris, 1990, V, 14 (267). 
ment, il multiplie non seulement les textes de lois (inflation législative) mais encore les principes (généraux), expression éminente des florissantes open textures en droit. Les uns et les autres participent au reste d'un même souci d'assomption de l'idiosyncrasie, avec des méthodes et une répartition des pouvoirs très différentes, de l'illusoire tout légal napoléonien (et l'Ecole de l'Exégèse) au tout prétoire volontairement investi de la charge du droit via l'usage dans les textes légaux des open textures et autres notions à contenu variable dont le législateur délègue la contextualisation au juge.

Mais la multiplication des principes généraux au libellé parfois fort lâche ne peut qu'augmenter le désarroi des hommes de loi face aux questions du sens et des valeurs. Elle induit un déplacement du centre de gravité substantiel du droit de la loi vers l'équité, bête noire des juristes modernes.

L'ars boni et aequi, l'art juridique (du bien et de l'équitable) semble l'emporter sur la science juridique dans la perspective de la pratique du droit, la perspective du «passage à l'acte» de la loi.

Mais la Rechtswissenschaft ne tiendra-t-elle pas sa revanche en enfourchant l'argument pluriséculaire de juge-arbitre donc arbitraire? A preuve, l'instrument privilégié du juge n'est-il pas l'équité, vecteur de tous les abus? Le juge n'est-il pas présumé sombrer dans l'arbitraire à la moindre parcelle de pouvoir concédée, image classique et combien erronée du juge («parlements») d'Ancien Régime, époque dont datent les expressions «bouche de la loi», «esclave de la loi» mais que l'on préfère attribuer à l'ère révolutionnaire pour mieux défendre et le monopole et la parfaite objectivité de la loi dont la complétude aurait éradiqué tout pouvoir normatif du juge, de la $\left\langle\right.$ juris-dictio» ${ }^{26}$ ?

Le retour de l'ars boni et aequi à la faveur de la prolifération des principes juridiques ne nous condamne-t-il pas au «gouvernement des juges » et de là au règne de l'arbitraire?

Reconnaître quelque pouvoir créateur ou normatif au juge oblige sans doute à quitter le domaine de l'objectivité, là où au sens littéral il n'y a que des Objets, monde pur de Sujets ou habité de manière éthérée par «Le» Sujet transcendantal, aussi parfaitement omniscient (en son ordre) qu'il est purement désincarné (donc «a-prudent»), territoire aussi rassurant qu'il était artificiel comme l'ont montré les philosophes des sciences en particulier ${ }^{27}$.

On ne rejoint toutefois pas nécessairement le monde de l'arbitraire selon cette logique binaire postulant que si un phénomène ne ressortit pas d'une pure objectivité alors il est ipso facto reconnu participer de l'arbitraire le plus crasse. Le praticien du droit sait d'expérience (pragmatiquement, à savoir via les pragmata) qu'entre l'Objectif, illusoire, et le Subjectif intégral - l'arbitraire - assez aisément reconnaissable et contre lequel le droit lutte depuis des siècles, s'étend l'espace de la subjectivité, de l'intersubjectivité précisément. Ce dernier s'éprouve structuré par une série d'attracteurs le gardant le plus souvent de l'arbitraire sans pour autant convoquer quelque croyance en l'objectivité.

${ }^{26}$ Sur ces points, voir notamment M.-F. Renoux-Zagamé, «Le «Royaume de la loi »: équité et rigueur du droit selon la doctrine des parlements de la monarchie », in Justices. Revue générale de droit processuel, Dalloz, Paris, N9, 1998, 17ss. (passim).

${ }^{27}$ Par exemple Popper; Serres; Prigogine/Stengers; D’Espagnat; Latour. 
En termes logiques, entre l'univocité (à la façon des faits qui parlent d'euxmêmes ou leur pendant juridique «la loi dit que», tous arguments d'autorité déguisés qui conduisent à prendre les mots pour les choses et à faire disparaître le Sujet) et l'équivocité (ne demeure cette fois que le Sujet en son bon vouloir qui ne suit que son bon plaisir), entre l'exclusivisme du parfaitement même et l'autisme du radicalement autre, il y a place pour le mélange, les hybrides, les mixtes de même et d'autre, des «entités» tissées de ressemblances et de dissemblances construites en référence à quelque modèle non d'obédience porphyrienne (classements dichotomiques en genre/espèces selon une structure arborescente) mais relevant d'une logique prototypique (ou encore encyclopédique), plus exactement la dialectique type/occurrence, exposée et mise en œuvre dans les travaux de Eco, lequel emprunte à C.S. Pierce et Aristote.

Eco fait ainsi justice de deux idoles. D'une part, le Veau d'or de l'univocité, figure du trop-plein, d'un principe - arche - si puissant que rien ne peut en sortir qui ne soit immédiatement jugé impropre, de l'ordre de l'accident (au sens philosophique du terme): sous les logiques de l'univocité opèrent toujours des métaphysiques de l'Un, d'inspiration néoplatonicienne ${ }^{28}$. D'autre part, la boîte de Pandore de l'équivocité, encore la figure du trop-plein, sans principe cette fois-ci - an-arche, anarchie par surabondance d'interprétations, chaos au sein duquel tout se vaut. Les deux idoles pèchent par manque de retenue, de mesure, par hybris à l'égard du contexte: ne lui rien prêter si ce n'est le dévoiement d'un sens pur déposé comme de toute éternité dans les mots mêmes du texte, comme le voudrait l'univocité, ou s'y complètement abandonner, en attendre tout jusqu'à faire du texte un simple «pré-texte».

Deux exclusivismes se dessinent ici sur la topologie sémiotique ternaire «morphosyntaxe [grammaire] - sémantique - pragmatique »: réduire la sémiotique à la seule sémantique $o u$ la réduire à la seule pragmatique. Mais qu'est-ce qu'une sémantique qui ne s'exerce pas, donc qui ne signifie rien, qui ne signifie pour personne? Et qu'est-ce qu'une pragmatique qui est pratique tout court, pratique de rien du tout: pratiquant quoi puisque rien ne lui préexiste? Autant d'exclusivismes, autant d'excès par quoi chacune des idoles témoigne de son tropplein (comme la normativité monolithique, sans différenciation que défendirent longtemps les juristes).

Où donc passe le sens, quel «mi-lieu» occupe-t-il entre la réification de l'univocité et la dissolution de l'équivocité?

Bobin décrit élégamment cette via media, guère discernable tant les idoles mentionnées ont fixé les esprits: «Je vais me glisser entre ces deux-là, entre la parole ivre et le Dieu taciturne. Il n'y a pas d'espace entre eux, aucune distance, mais je saurai bien me faufiler, donner à mon âme la minceur qui convient, l'humilité nécessaire. $\gg^{29}$ Notre position sera humble, celle de quelques frontières tracées, floues et mouvantes, bien davantage limites esquissées que fixées, décrétées. Là, dans le «plus ou moins » ou «le plus fréquemment» se situent les Limites de l'interprétation de Eco.

${ }^{28}$ Voir concernant Porphyre (et ses arbres), Alain de Libera, La querelle des universaux. De Platon à la fin du Moyen Âge, Seuil, Paris, 1996, passim, en particulier 44.

${ }^{29}$ C. Bobin, Le Très-Bas, Gallimard, Paris, 1992, 65. 
Le droit participe immédiatement, plus, éminemment, de cette problématique comme annoncé par P. Valéry qui «au cours d'une conférence faite à l'Ecole des Sciences politiques, observait que ce qui manque aux nombreuses définitions du Droit, présentées par les meilleurs auteurs de Justinien à Kant, c'est la mention du langage, qu'il n'est pas possible pourtant de séparer de la notion de Droit.» ${ }^{30} \mathrm{On}$ s'étonnera à juste titre de ce que le juriste témoignerait une attention si peu soutenue à ce dont il se sert en tout premier lieu: la langue. Etonnement d'autant plus grand qu'est aujourd'hui largement reconnue «l'importance capitale du débat sur la langue et les autres systèmes de signes pour éclaircir nombre d'autres problèmes, de l'éthique à la métaphysique.» ${ }^{31}$

L'assomption de l'idiosyncrasie non seulement s'inscrit légitimement dans cet ordre de difficulté mais en a peut être constitué la difficulté originelle, le questionnement matriciel: des procédés déictiques à la base de tout langage naturel jusqu'aux discussions toujours actuelles parce qu'indépassables, problématique ramassée dans la formule philosophique de «querelle des universaux ».

Sans doute faut-il encore préciser ici que sémiotique et herméneutique constituent les lieux privilégiés d'assomption du langage dans une dimension non exclusivement linguistique mais plus fondamentalement cognitive, comme vecteur de la catégorisation, et par là médium inéluctable de la qualification, celleci constituant le lieu même de la construction de la commensurabilité de la loi et du cas par quelque indispensable arbitre ou juge aux fonctions d'Hermès, tiers, medium.

Et si le désarroi subsiste, sans doute est-ce pour bonne part dû à cette quête incessante de l'unité, le retour à l'Un des métaphysiques néoplatoniciennes. Peutêtre pourrait-on avancer cette hypothèse de lecture téméraire pour Devant la loi: plus on remonte les hypostases en direction de l'Un (la réduction à l'Un, au pointorigine), plus elles deviennent puissantes, «absorbantes», comme la succession de gardiens toujours plus puissants, jusqu'à l'Un, la Loi qui absorberait le singulier et donc ne le considérerait pas en lui-même, en son idiosyncrasie.

Cette aspiration irrépressible au monde clos, propension naturelle à l'unicité ${ }^{32}$, s'avère à ce point irrésistible qu'en elle se fonde la science moderne «classique» et la foi dont elle est l'objet ${ }^{33}$. Or, la science moderne n'est pas armée cognitivement pour appréhender le complexe et le «systémique», notamment en postulant une équivalence entre univocité, unité et universalité, écartant de la sorte toute médiation constitutive du phénomène à analyser par le ministère du contexte et de l'interprète «observateur».

${ }^{30}$ R. Nerson, «Exercices de vocabulaire», in Mélanges offerts à Monsieur le Professeur Pierre Voirin, LGDJ, Paris, 1967, 604.

${ }^{31}$ U. Eco, Sémiotique et philosophie du langage, PUF, Paris, 1988, 10.

${ }^{32} \mathrm{~J}$. Lenoble/F. Ost, Droit, mythe et raison. Essai sur la dérive mytho-logique de la rationalité juridique, Publications des facultés universitaires Saint-Louis, Bruxelles, 1980, après avoir rappelé, à la suite de Kant, «la propension de l'esprit humain à introduire l'unité dans la connaissance du multiple» (119), nous avertissent que «[1]e danger est grand cependant de prendre cette unité d'une intelligence suprême pour déjà réalisée et, par un funeste renversement, céder à la raison paresseuse [...] qui nous fait tenir la perfection finale pour fondement réel de la réalité.» (120)

${ }_{33}$ Voir Prigogine/Stengers, op. cit., spécialement le Livre I «Le mirage de l'universel: la science classique». 
En bref, sa métaphysique (ou épistémologie) s'articule autour du référent unique et exclusif de l'essence, considérant corrélativement les existants, les pragmata comme des «accidents», quelque chose qui «accidit», qui se surajoute, véritable «choix philosophique: la prééminence accordée à l'être sur le devenir, à la permanence sur le changement $»^{34}$, à la conséquence funeste pour l'assomption de l'idiosyncrasie: «ce que la science classique touche se dessèche et meurt, meurt à la diversité qualitative, à la singularité, pour devenir la simple conséquence d'une loi générale.» ${ }^{35}$

Eco, à son tour, a vigoureusement dénoncé cette mainmise de l'analytique sur les conceptions modernes et contemporaines de la cognition (du connaître/ comprendre). Si la sémiotique ${ }^{36}$ a emprunté aux langues naturelles ses notions et articulations nodales ${ }^{37}$, on ne saurait toutefois l'y réduire sans s'abîmer dans une métaphysique de l'univocité, une structure de pensée binaire qui juge sans appel arbitraire toute catégorie aux frontières non discrètes ou toute qualification opérant sur le mode d'une logique du «plus ou moins», «différentielle»: «le modèle du signe linguistique s'impose graduellement comme le modèle sémiotique par excellence.

Mais cette conclusion [...] débouche sur un modèle linguistique qui est désormais cristallisé dans sa forme la plus «plate», celle qu'entérinent les dictionnaires et, malheureusement, une bonne partie de la logique formelle ${ }^{38}$. La conception monolithique de la normativité doit beaucoup à cette vision analytique du réel.

Pourtant, le locuteur occidental affirme (statistiquement parlant) que l'aigle est plus oiseau que la poule. Ses catégories mondaines ne sont pas homogènes: pour appréhender le «réel», il compare, hiérarchise, différencie («gradualise»), juge bien davantage qu'il ne calcule.

Le scientisme, en revanche, se rabat sur les analyses componentielles. Dans ce type d'approche, le signe est reçu dans les formes d'une entité statique biface signifiant/signifié, le signifié étant intégralement donné dans un nombre fini de traits sémantiques pertinents ou primitif ${ }^{39}$. Ces derniers sont imaginés à l'abri des influences de la pragmatique, franc d'instructions contextuelles en particulier. L'analyse componentielle tient donc dans l'inventaire des primitifs censés épuiser la réalité de l'entité sujette à interrogation.

Le scientisme renoue de la sorte avec les arbres de Porphyre - en termes sémiotiques le signe-équivalence -, les divisions binaires au service de l'univocité et de ses ressorts: plate mimésis et stricte «con-formité». On se rappellera alors que «forme» vient de eidos, idée, forme (cause formelle dans la philosophie grecque), donnant aussi bien formalisme qu'idéalisme.

${ }^{34}$ Idem, 375.

35 Idem, 94.

${ }^{36}$ «Théorie générale des modes de signifier, science générale des signes (syn. SEMIOLOGIE)», Dictionnaire de la langue française, lexis, Larousse, Paris 1988, verbo SEMIOTIQUE.

${ }^{37}$ Voir Eco, Sémiotique, 42 ss.

${ }^{38}$ Eco, Sémiotique, 43.

${ }^{39}$ U. Eco, Les limites de l'interprétation, Le livre de poche, Paris, 1994, 360, les définit de la manière suivante: «expressions métalinguistiques qui ne sont pas des mots en soi et qui ne nécessitent aucune interprétation ultérieure.» 
Faire de la «con-formité » le critère du juste, du droit revient à dépouiller l'occurrence sur laquelle on l'applique de toute sa singularité: elle n'est pertinente pour le droit que sous l'angle des primitifs ou des propriétés arrêtés dans le texte de loi $^{40}$, le point de vue de la structure l'emportant alors sur celui de la fonction. On a donc perdu le contexte et par là perdu la pragmatique, confirmant la conception essentialiste ou réifiante du positivisme juridique, cette expression du scientisme en droit. Rigaux, cruel, parle des «vaches sacrées de la philosophie spontanée des juristes, la recherche du sens littéral, la théorie de l'acte clair, le devoir du juge d'obéir à la loi, la distinction entre le fait et le droit [...], l'idée que pour toute question litigieuse il n'existerait qu'une seule solution correcte. Ces lieux communs de la pensée juridique participent à une taxinomie binaire, c'est-àdire à une méthode de raisonnement traçant une démarcation rigide entre la face positive et la face négative de toute réalité.» ${ }^{41}$

L'analyse componentielle doit s'entendre littéralement comme participant d'un paradigme analytique, pour lequel «le tout n'est que la somme des parties », ce dont témoigne au premier chef la description en un nombre fini de traits sémantiques (monde clos), sous forme de primitifs. «Le malheur c'est que (i) il n'existe pas de critère pour établir si un trait est analytique ou synthétique; (ii) toute tentative pour identifier un bloc d'universaux sémantiques s'est toujours limitée à de petites portions du lexique; (iii) enfin une représentation dictionnairique n'explique pas pourquoi le locuteur est à même de comprendre les énoncés formulés dans sa langue. $\rangle^{42}$ On comprend que dans pareil paradigme, bien reçu dans la sphère juridique, le «fait» et le «droit» se muent en «des entités réifiées $»^{43}$.

\section{TITRE 3. \\ ESQUISSE D'UNE PHILOSOPHIE RELATIONNELLE («ANALOGIQUE») DU DROIT}

Eco propose un autre modèle de représentation sémantique, proprement sémiotique celui-ci, l'encyclopédie ${ }^{44}$, laquelle assume l'épaisseur diachronique d'une culture et de sa langue, dont elle constitue, en quelque manière, la «bibliothèque idéale $»^{45}$. Elle «S'est constituée à travers l'exercice de cette langue ${ }^{46}$ et comprend «les conventions culturelles que cette langue a produites et l'histoire

${ }^{40}$ En vérité, découlant de l'interprétation du texte légal et non de celui-ci comme tel.

${ }^{41}$ F. Rigaux, La loi des juges, Odile Jacob, Paris, 1997, 8.

${ }^{42}$ U. Eco, Le signe, Le livre de poche, Paris, 1992, 147.

${ }^{43}$ Rigaux, op. cit., 48.

${ }^{44}$ L' «encyclopédie » intègre le principe d'interprétance, écartant par là toute possibilité d'analyse finie d'un terme: l'encyclopédie, pénétrée du processus illimité de la sémiosis, enregistre les interprétants et les interprétants d'interprétants, renvois sans terme final et passant souvent d'un type de sémie à l'autre (d'un mot de la langue naturelle à une image, à un symbole, à un son, à un phénomène naturel pris comme indice, à un schéma, etc.).

${ }^{45}$ Eco, Sémiotique, 110.

${ }^{46}$ Eco, Les limites de l'interprétation, 133. 
des interprétations précédentes de nombreux textes, y compris le texte que le lecteur est en train de lire en ce moment. ${ }^{47}$

La mention de «l'exercice de cette langue» pointe la nature dynamique de cette manière de concevoir le signe et partant le réel. Et les termes d' 'exercice» et de «dynamique» sont, à leur tour, des expressions traduisant l' «acte» aristotélicien (du couple puissance/acte) et de là le «passage à l'acte» qui manque si cruellement dans Devant la loi. On se trouve aux antipodes d'une ontologie statique, une ontologie réduite aux essences, entités éminemment réifiées. Rien ne semble plus contraire à une métaphysique polaire que la considération des usages, des pratiques, des pragmata, lesquelles, dans un paradigme essentialiste, résonnent comme de simples accidents de l'essence, seule vérité authentique à la manière des Idées platoniciennes. Dans la perspective d'Aristote, au rebours, les existants (pragmata), les usages sont la perfection ou achèvement des essences, celles-ci n'étant au reste connaissables qu'à partir de ceux-là.

Traduite dans la sphère juridique, cette dernière considération accrédite un sens de la loi véritablement connu - « véritablement» entendu dans la lumière de la justice - que dans et à travers ses applications: les occurrences rétroagissent nécessairement sur le type (légal) qui ne fait que fixer le cadre des applications. La loi ne devient donc accessible que par le ministère de quelque juge ou arbitre qui, littéralement, meut le droit dans le cas soumis, exerce («acte» ou actue) la justice in casu.

Le gardien, au contraire, étouffe tout mouvement, laissant la loi dans une posture hiératique et l'homme de la campagne dans l'«in-justice», une loi qui ne passe pas à l'acte et même qui l'ignore.

Sans doute le droit a-t-il toujours opéré dans la perspective de l'acte: «rendre justice». En revanche, sa théorie dans l'ère moderne s'est rarement aventurée du côté des pragmata comme lieu où se «par-fait», se fait de part en part, lieu de perfection du droit, comme si la théorie craignait quelque adultération en devenant la théorie de la pratique effective du droit plutôt que celle de l'Idée du droit. Les Anciens, pénétrés de rhétorique se gardèrent du chant des sirènes de la Rechtswissenschaft et aucun Napoléon ne leur annonça la fin du droit. Le paradigme analytique cartésien a désormais atteint ses limites, y compris en droit, le structuralisme est trop «compliqué » et pas assez «complexe».

La lecture des textes légaux actuels rend ce dépassement patent. La multiplication des open texture et des références aux principes généraux rend indispen-

${ }^{47}$ Eco, Les limites de l'interprétation, 133. Aussi un chat est-il, d'un point de vue encyclopédique, non seulement «un félin domestique, mais c'est aussi l'animal défini par les classifications zoologiques comme felis catus, l'animal adoré par les Egyptiens, l'animal qui figure dans l'Olympia de Manet, l'animal qu'on mangeait comme un morceau de roi dans le Paris assiégé par les Prussiens, l'animal chanté par Baudelaire, l'animal que Collodi associe au renard pour la ruse et la méchanceté, [...], l'animal paresseux qui s'attache aux lieux mais ne va pas mourir d'inanition sur la tombe de son maître, l'animal de prédilection des sorcières, etc.: tout cela sont des interprétations de l'expression «chat<. Elles sont toutes enregistrées, posées intersubjectivement dans quelque texte de cette immense bibliothèque idéale dont le modèle théorique est l'encyclopédie. Chacune de ces interprétations définit sous quelque rapport ce qu'est un chat» (Eco, Sémiotique, 109-110). Eco précise: «Chacune d'entre elles est valable et actualisable dans un contexte déterminé, mais l'encyclopédie devrait fournir idéalement des instructions permettant d'interpréter de la manière la plus fructueuse l'expression «chat< dans de nombreux contextes possibles.» (ibidem) 
sable l'adoption d'une perspective dynamique dans l'étude du phénomène juridique, perspective non «formaliste», au sein de laquelle les applications redeviennent «destinales »: le droit est en premier lieu une praxis; au juge est fait, de par la loi même, obligation de trancher sous peine de déni de justice; la cause (causa) doit pouvoir devenir chose (causa) dans la lumière de la ratio legis (la «raison» de la loi, mieux la ratio juris) qui indique l'orientation de cette transmutation. Alors la cause finale, la téléologie, fait retour en la sphère juridique et le passage de la cause à la chose recouvre une position éminente, centrale.

La loi-forteresse de la fabula kafkaïenne campe le modèle mortifère de la loi pour la loi c'est-à-dire un droit qui non seulement est réduit à son expression formelle (la loi), à savoir un droit prenant l'un de ses moyens pour sa fin mais pis, un droit qui a perdu sa finalité, trop modeste peut-être pour la morgue de l'homme moderne jaloux de l'illusion de son illimitation. Car le droit est tout fait de limites, plus exactement de délimitation qui est l'action de délimiter, et il s'emploie depuis la nuit des temps à opérer des partages, à l'enseigne des harpédonaptes.

Aux temps de l'Egypte pharaonique, en effet, les crues du Nil apportaient la plus parfaite «con-fusion»: fusionnaient ensemble les terres, effaçaient les frontières, instaurant un complet mélange. Les autorités en appelaient alors à l'intervention, à la médiation, des scribes-géomètres afin (à fin, finalité) de redistribuer les surfaces, redéfinir les partages par où droit et géométrie semblent partager une origine commune: «Les Egyptiens [...] avaient pris pour juges de leurs contentieux de bornage ceux qui savaient obtenir les superficies par des opérations sur les longueurs, par le cordon, l'unité, la mesure, l'écriture et le prestige: voilà les harpédonaptes, les premiers des géomètres.» ${ }^{48}$

Délimiter prend ici tout son sens: le juriste-géomètre est plongé dans le concret du limon, dans la concrétion de l'humus, la confusion du déluge. Et au sein des pragmata, il tente de distinguer, de départager le «mien» et le «tien» (suum cuique tribuere). Il agit dans le mélange, dans l'hybride, médiatise le confus et tente de le polariser en créant le lien qui fonde la reconnaissance mutuelle au principe du droit, institutionnalisant le «symbole»: «Qu'est-ce qu'un acte notarié à côté de la demeure où l'on habite? Comment comparer cette fragile feuille de papier à l'épaisseur des murs et des mémoires? Aucun rapport de ressemblance, aucun mimétisme, aucune référence, aucun plan. Et pourtant, en cas de conflit, en cas d'héritage, en cas de dispute, c'est bien par le lien foudroyant de cette feuille dérisoire avec le corpus des textes, par l'intermédiaire des avocats et des juges, que je pourrai prouver, authentifier mon bien - et conserver ma maison. L'attache est minuscule et pourtant totale; l'emprise infime et pourtant capable de se lier à tout le reste.» ${ }^{49}$

Le droit se donne ici à la manière d'un symbole «maximal» puisqu'il est convoqué dans sa totalité, comme système juridique, dans chaque cas singulier: l'occurrence fait parcourir l'ordre juridique dans son ensemble et «dans l'entier de sa complexité » ${ }^{50}$, lien «intégral » comme celui que révèle la sémiotique de Eco dans la forme systémique de l'encyclopédie. Présence «foudroyante » qui ouvre la

\footnotetext{
${ }^{48}$ M. Serres., Les origines de la géométrie, Flammarion, Paris, 1993, 49-50.

49 B. Latour, La fabrique du droit, La Découverte, Paris, 2002, 284.

${ }^{\text {so }}$ P. Moor, Pour une théorie micropolitique du droit, Paris, PUF, 2005, 15.
} 
voie vers l'assomption de l'idiosyncrasie: c'est par la totalité que s'éclaire la singularité, pensée proprement systémique ou logique de réseau, logique réticulaire tissée de rétroactions dont participe éminemment l'encyclopédie sémiotique dont le système juridique n'est qu'une portion, singulière sans doute.

La prise en considération de l'exercice, savoir des usages, des pratiques dans l'élaboration des catégories cognitives transforme leur structure: à l'arborescence (arbre de Porphyre) se substitue une représentation en réseau, elle-même articulée autour d'un noyau de catégorie, mixte de sémantique et de pragmatique, sorte d'occurrence prototypique, en référence à laquelle se conduit le jugement d'analogie permettant d'attribuer (temporairement) le cas à la catégorie. L'opération complexe de comparaison de l'occurrence candidate avec l'occurrence prototypique (légale) en vue de sa qualification s'articule sur un mode de raisonnement ne ressortissant pas de la déduction ou du syllogisme mais de l'abduction.

On a pu définir l'abduction comme une «Démarche logique (au sens large) hypothétique (essai/erreur) consistant, face à un résultat («fait») surprenant, à inventer ou découvrir simultanément une règle et un cas, de telle sorte que si le résultat est bien un cas de la règle, alors il ne présente plus rien de surprenant: il est apprivoisé ${ }^{51}$. L'abduction, en simplifiant à l'extrême, se modélise comme une opération inductive sous hypothèse, une comparaison se développant entre occurrences, bottom-bottom, entre l'occurrence modèle posée par la loi (le prototype légal) et l'occurrence litigieuse.

Nous trouvons sous la plume de Ricœur la description la plus didactique de ce que peut être l'abduction dans le champ du droit et le changement de paradigme juridique qu'elle initie: «L'application d'une règle juridique est en fait une opération très complexe où l'interprétation des faits et celle de la norme se conditionnent mutuellement. Du côté des faits de la cause en procès, le même enchaînement factuel peut être interprété de plusieurs manières [...]; et telle façon d'en lire l'enchaînement est déjà orientée par la présomption selon laquelle tel enchaînement narratif place le cas considéré sous telle règle de droit. Quant à la règle de droit, elle présente elle-même une open structure qui la met sous la dépendance des interprétations antérieures adoptées dans des cas semblables; mais l'idée même de cas semblables, présupposée par la règle du précédent ${ }^{52}$, résulte de l'interprétation du degré de ressemblance ou de dissemblance avec les cas précédents. [...] Cela dit, il n'y a plus rien de scandaleux à avouer le caractère vague du langage juridique, les conflits entre normes, le silence de la loi sur les cas inédits, la nécessité de choisir entre la lettre et l'esprit de la loi: c'est en l'appliquant que non seulement on reconnaît la norme comme contraignante, mais que l'on fait l'épreuve de sa variabilité, et que s'exerce le jeu décrit plus haut de l'interprétation mutuelle entre la loi et les faits. $\nu^{53}$

51 A. Papaux, op. cit., 523.

${ }^{52}$ Y. Gaudemet, «Fonction interprétative et fonction législative: aménagements juridiques de leurs rapports », in Interprétation et droit, Bruylant, Bruxelles, 1995, 201 ss (204) parle de « souscription implicite (ou expresse) de tout système juridictionnel à la règle du précédent », en laquelle se fonde l'autorité de l'interprétation du juge. La règle du précédent n'opère donc pas dans les seuls pays de common law. Dans le même sens, Rigaux, op. cit., 129.

${ }^{53}$ P. Ricœur, «Le problème de la liberté de l'interprète en herméneutique générale et en herméneutique juridique», in Interprétation et droit, Bruylant, Bruxelles, 1995, 177ss. (180). 
L'abduction mélange le fait et le droit, parce qu'elle procède d'une philosophie de la relation et non des pôles. Elle se passera donc de gardiens. Son problème ne sera pas de rapprocher des entités initialement séparées, à tout jamais peut-être, en multipliant les intermédiaires, vision artificielle et typiquement moderne du réel selon Latour qui l'a durement critiquée dans Nous n'avons jamais été modernes. On est et naît dans le mélange, le mixte et le travail cognitif tient davantage dans la distinction, la purification, la mise en ordre, l'élaboration de la mesure commune (la «com-mensurabilité») et donc la comparaison construite que dans la constatation objective et désincarnée de l'observateur neutre.

Le corpus théorique nécessaire pour pénétrer plus avant ces idées est colossal. Une philosophie de l'acte qui, on l'a vu, n'est pas un essentialisme mais ne saurait être non plus un «existentialisme»: le passage à l'acte de la puissance constitue le moment nodal. Une philosophie de la reconnaissance que Ricœur a développée comme telle à la fin de sa vie ${ }^{54}$. Une sémiotique qui embrasse ces deux moments philosophiques, une sémiotique dynamique élaborée sur fond de signe-renvoi (d'interprétants en interprétants), au rebours du traditionnel signe-équivalence (signifiant/signifié), qui maintienne la relation de reconnaissance tout au long du processus de la sémiosis, du parcours de l'encyclopédie. Ces diverses instances de la pensée devront encore prendre chair en droit, plus exactement y être apprivoisées car elles y sont couramment pratiquées mais rarement théorisées, que l'on pense au lien foudroyant de l'occurrence avec le système juridique dans son entier ou aux notions de prudence et d'équité. A la faveur de ces dernières se reconnaît la présence inexpugnable de la subjectivité, la médiation nécessaire, intrinsèque du Sujet, juge ou arbitre, et plus souvent encore citoyen pour la grande part du droit s'exerçant à satisfaction hors tribunaux. Au mieux ouvrons-nous la porte mais Devant la loi ne nous autorise point à franchir le seuil: il y avait lieu d'expliciter l'insuffisance, l'absurde, la panne de la Loi-forteresse ou forteresse-loi. Et si « une glorieuse lumière [qui] jaillit éternellement de la porte de la loi » (Kafka), il sera encore temps d'attendre, en souffrance de quelque autre étude du versant «positif».

\section{CONCLUSION}

C'est bien cette mise en dialogue, cet ajustement réciproque du fait et du droit, du cas et de la loi, savoir la construction de la commensurabilité qui n'advient pas dans Devant la loi. Le passage à l'acte aurait-il pu se réaliser, la loi assumer l'idiosyncrasie? Kafka ne semble pas vouloir nous en offrir l'espoir. Après l'attente d'une vie, à la manière d'un chevalier quêtant le Graal, mais dans une veine absurde (à l'enseigne de la prière aux puces), l'homme mourant quand la porte devient $s a$ porte.

Epuisement d'un paradigme tout aussi bien: les cas d'application ne sont pas que des accidents du droit (au préalable réduit à la loi) mais son étoffe même, sa fin, son but: la loi est faite pour l'homme et non l'homme pour l'institution, et le

\footnotetext{
${ }^{54}$ P. Ricœur, Parcours de la reconnaissance, Stock, Paris, 2004.
} 
droit pour le cas singulier, dût-il en appeler à l'équité, au jugement prudentiel, à l'abduction pour «dire le droit» (jurisdictio).

Aussi la noblesse de la loi sera-t-elle moins dans la hauteur de ses principes, trop prompts à la grandiloquence, que dans les médiations qu'elle organise. Quelle que soit la finesse des médiations assumées, il est à douter que le droit puisse, comme institution, répondre à l'appétence de justice et de reconnaissance qu'éprouve l'individu plongé dans un univers de juxtaposition, d'atomes sociétaux, attente, prima facie pragmatique, plus gravement symbolique et ultimement ontologique: «tu es insatiable» dit le gardien.

Université de Lausanne 